\title{
COPD treatment: about collateral channels and collapsing airways
}

\author{
Ralf-Harto Hubner and Dominik Herzog
}

Affiliation: Dept of Internal Medicine/Infectious Diseases and Respiratory Medicine, Charité Universitätsmedizin Berlin, Berlin, Germany.

Correspondence: Ralf-Harto Hubner, Dept of Internal Medicine/Infectious Diseases and Pulmonary Medicine, Charité - Universitätsmedizin Berlin, Augustenburger Platz 1, 13353 Berlin, Germany.

E-mail: ralf-harto.huebner@charite.de

@ERSpublications

Dynamic airway collapse during Chartis doesn't identify the presence of collateral channels in that specific lobe http://ow.ly/YGV0G

Collateral channels inside the human lungs? This phenomenon, first described by KoHN [1] in 1893, is the subject of recent investigations in the field of endoscopic lung volume reduction (ELVR) for the treatment of severe chronic obstructive pulmonary disease (COPD). ELVR with valves is a new and promising therapeutic option for irreversibly damaged emphysematous lungs. The aim of the therapy is the complete occlusion of a lobe, with endobronchial one-way valves delivered through a bronchoscope to intentionally induce a targeted atelectasis of the most damaged lobe. Many studies have described improvements in lung function, quality of life and exercise capacity in a subset of severe COPD patients, where interlobar collateral ventilation was an exclusion criterion for valve treatment [2-8]. Interlobar collateral ventilation is defined as the ventilation of alveolar structures through passages or channels that bypass the normal airways, and includes the interalveolar pores of Kohn, the bronchiole-alveolar communications of Lambert and the interbronchiolar pathways of Martin [9-14]. Collateral ventilation is present not only in COPD lungs, but also in healthy lungs [15]. The physiological function of the collateral channels is still unknown; in COPD patients they might provide channels of low resistance to better ventilate less emphysematous lung segments. The importance of collateral ventilation was ignored for over a century, but now, with their role in ELVR and the development of new bronchoscopic techniques, the study of these little channels has gained enormously in importance.

It has become clinical routine that collateral ventilation of the target lobe must be assessed prior to valve treatment, e.g. by physiological testing using an endobronchial in vivo measurement system called "Chartis" (Pulmonx Inc, Redwood City, CA, USA) or by anatomical analysis of lung fissure integrity from computed tomography (CT) scans. The first major valve trial with 321 patients, published in 2010 [2], revealed in a retrospective ad hoc analysis the importance of fissure completeness in ELVR treatment: while patients with incomplete fissures based on visual high-resolution CT analysis improved their forced expiratory volume in $1 \mathrm{~s}$ (FEV1) by only $2.0 \%$ from baseline to 6 months, the subgroup with complete fissures achieved significantly better clinical outcomes and an FEV1 increase of $16.2 \%$. The most important problem concerning visual fissure analysis on CT scans is that this technique is highly operator dependent. KoENIGKAM-SANTOS et al. [16] found that pulmonologists and radiologists agree at best moderately in visual fissure analysis, and concluded that visual analysis might require experienced chest radiologists.

The Chartis (Pulmonx Inc) technology was introduced into clinical practice in 2009. This helped to objectively assess collateral ventilation and thus became clinical routine [17-19]. A recently published trial prospectively confirmed the importance and efficacy of Chartis in selecting adequate patients for ELVR and that valve therapy can benefit a broad range of advanced stage emphysema patients [20].

Received: Feb 152016 | Accepted: Feb 162016

Conflict of interest: Disclosures can be found alongside the online version of this article at erj.ersjournals.com

Copyright OERS 2016 
However, Chartis assessments and measurement interpretation may sometimes be challenging when Chartis patterns cannot be attributed directly to the presence or the absence of collateral ventilation. The publication in this issue of the European Respiratory Journal by GESIERICH et al. [21] describes the interesting and still little understood Chartis pattern called the "collapse phenomenon", which is also known as the "low flow" or "no flow" pattern in the literature [22, 23]. This pattern has been recently characterised as a sudden decline in expiratory flow towards zero, a sharp increase of the resistance curve to over 100 and a total exhaled volume $<50 \mathrm{~mL}$ (figure 1a) [24].

GESIERICH et al. [21] postulate that a collapse of the distal airways is an explanation for this Chartis pattern, which is reasonable although the causes are still elusive [22-24]. How and why could an airway collapse occur only during Chartis assessments? In healthy lungs, smooth muscle tone keeps the airways open, preventing their collapse. However, in severe emphysema, characterised by obstructed distal airways and loss of elastic recoil, expiration becomes an active process of pushing air through the airway obstruction. Thus, the pressure in the obstructed airways decreases, leading to an airway collapse according to the Bernoulli principle (figure 1b) [25]. It is possible that the Chartis assessment may augment the Bernoulli
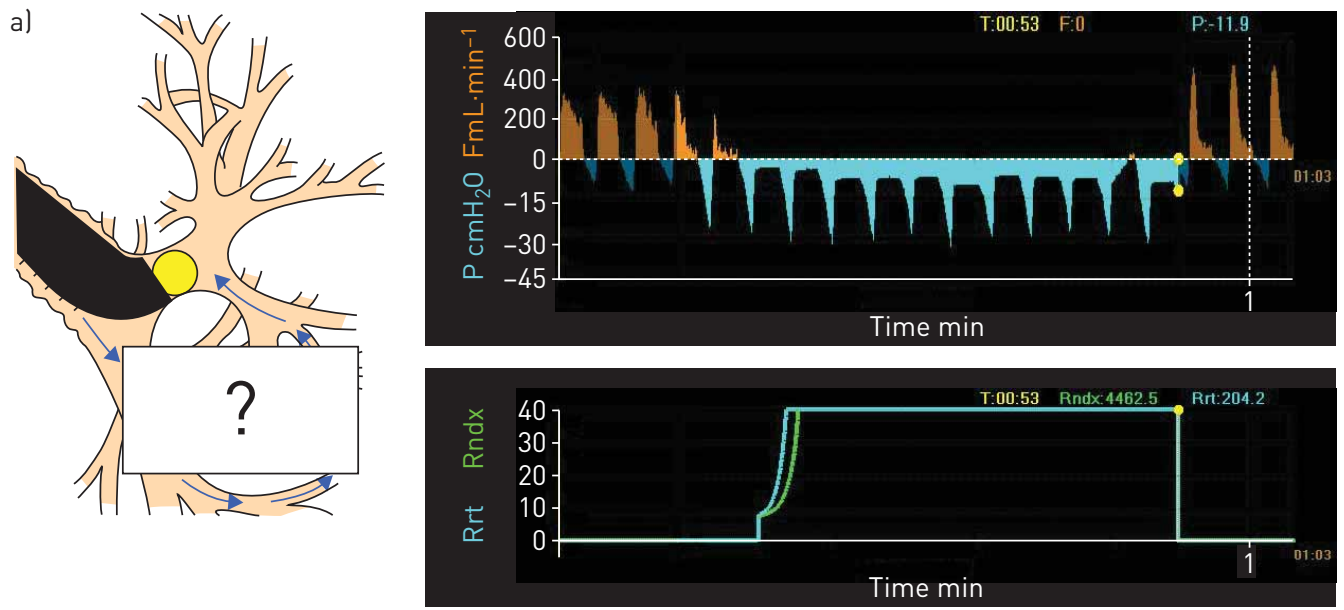

b)

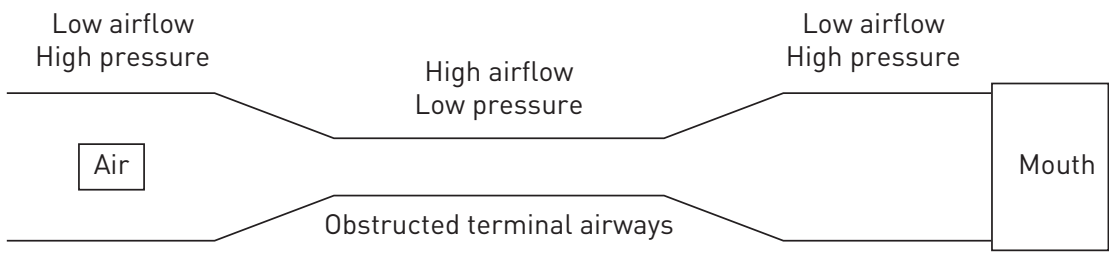

c)

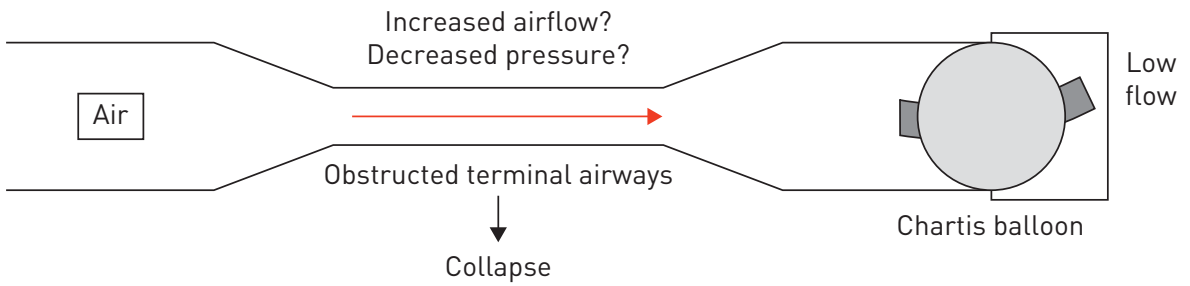

FIGURE 1 a) Example of collapse phenomenon or low flow in Chartis (Pulmonx Inc) assessment. The graphic on the left illustrates the Chartis assessment: the Chartis catheter is inserted into the bronchus of the target lobe via the working channel of the bronchoscope. An inflatable balloon is located at the tip of the Chartis catheter, designed to seal the airway. Sensors located in the external console measure the air that is expired by the patient. The question mark symbolises that the collapse phenomenon is uninformative regarding collateral ventilation status. The graphs on the right show the corresponding results on the Chartis console: a sudden decrease of expiratory flow (orange) to zero and an abrupt increase in resistance index (blue and green). b) Bernoulli's principle in obstructed airways. In severe emphysema, expiration is an active process of pushing air through the airway obstruction. As a consequence, the airflow increases as the pressure decreases in the obstructed airways (modified from [32]). c) Possible pathophysiology of the collapse phenomenon in Chartis assessments. At the beginning of Chartis assessment (grey balloon) airflow may be increased in these obstructed airways (red arrow) leading to a decreased pressure so that a sudden functional bronchial collapse occurs. 
effect because airflow might be increased in these obstructed airways, and this would lead to a decreased pressure beyond the threshold so that a sudden functional bronchial collapse during expiration at the beginning of the Chartis assessment occurs irrespective of collateral ventilation status (figure 1c). It would be interesting to find out whether CT scans during Chartis assessments could detect local air trapping in assessed lobes as an indirect sign of small airway collapse. In addition, little is known about the collapse phenomenon in healthy subjects. These issues should be addressed in further studies.

Not knowing the exact physiological causes, why do we pay so much attention to this phenomenon? To start with, it is a frequent Chartis pattern; GesIeRICH et al. [21] found it in about one third of all their Chartis assessments. Moreover, the collapse phenomenon is predominantly attributed to the lower lobes, suggesting a heterogeneous distribution of airway instability in the human lung, a new and exciting finding that fits with the concept of COPD heterogeneity. In COPD, phenotypes have been defined with regard to clinical, physiological and radiological manifestations, as well as exacerbations, inflammatory characteristics, patterns
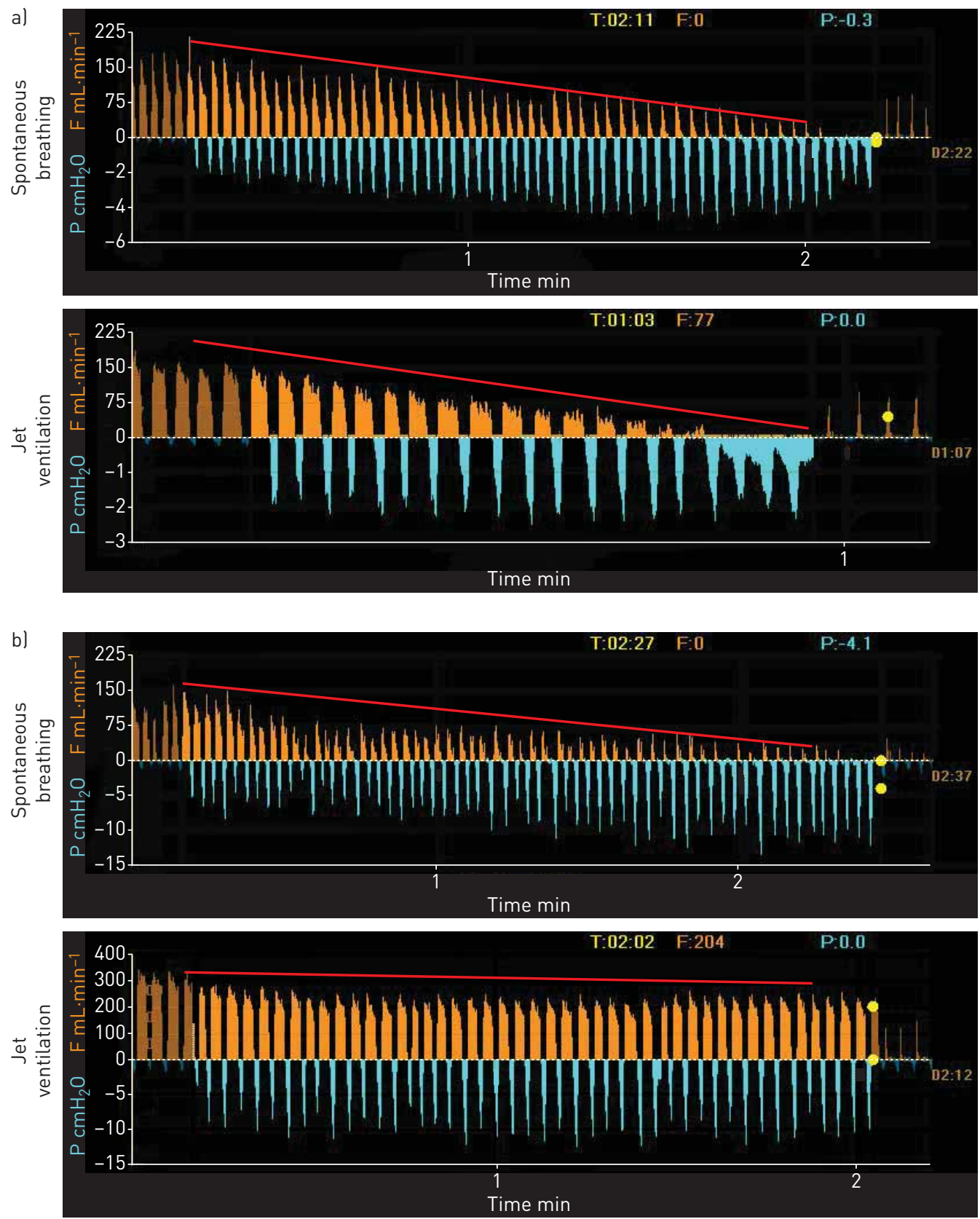

FIGURE 2 Examples of a) concordant and b) discordant results of Chartis (Pulmonx Inc) assessments under spontaneous breathing and jet ventilation. The red line symbolises expiratory flow. 
of comorbidities and now, in addition, heterogeneous airway instability [26]. This subdivision of COPD into phenotypes is fundamental to individualise and optimise treatment options for COPD patients [27].

Another very interesting observation is that the collapse phenomenon occurs almost twice as frequently under jet ventilation than under spontaneous breathing. Since the collapse phenomenon is an inconclusive Chartis pattern that does not provide valid information about the collateral ventilation status, GESIERICH et al. [21] recommend performing Chartis assessments only under spontaneous breathing in order to minimise the frequency of the collapse phenomenon. However, from our experience, applying both techniques we have often seen matching results (figure $2 \mathrm{a}$ ), but have also observed that in some patients collateral ventilation negative Chartis results under spontaneous breathing became collateral ventilation positive with jet ventilation (figure $2 \mathrm{~b}$ ). Should such a patient be treated with valves? It is possible that micro-collateral channels, undetectable with Chartis under spontaneous breathing, may open with jet ventilation. Nevertheless, we do not know if these hypothesised micro-collateral channels are clinically relevant and thus impose an effect on ELVR. To date, there is no study data available to compare spontaneous breathing or jet ventilation with respect to the clinical outcomes. Maybe using both might help to better select appropriate patients for valve therapy, using jet ventilation primarily and then, if the collapse phenomenon occurs, the target lobe might be measured again under spontaneous breathing.

Interestingly, GESIERICH et al. [21] complemented and compared the collateral ventilation Chartis analysis with visual analysis of fissure integrity, although there are also software-based automatic quantitative assessments of CT scans (QCT) that are less operator dependent [28]. The accuracy to predict clinical outcomes were 79\% with QCT and 76\% with Chartis in another study [29] and the clinical outcomes of studies using Chartis technology and QCT have also been shown to be similar $[8,30]$, thus confirming a strong relationship between fissure morphology and functional measurements of collateral ventilation [31]. While Schunmann et al. [29] observed one third of subjects with discordant collateral ventilation status, Gesierich et al. [21] found discordant collateral ventilation status in only $10.7 \%$. The low case numbers and lack of clinical outcomes make it difficult to draw conclusions whether Chartis or QCT can better predict clinical outcomes, and this will be a subject for future studies. Both techniques have advantages and disadvantages and the present publication considers both as complementary assessment procedures.

For centres working exclusively with Chartis, the following question arises: since the collapse phenomenon is an inconclusive and frequent Chartis pattern, which is uninformative with respect to the presence or absence of collateral ventilation, can patients with a collapse phenomenon lobe be successfully treated with valves? Gesierich et al. [21] reported 18 patients with a collapse phenomenon in the target lobe that were treated with valves, comprising 15 patients with complete fissures and three patients with incomplete fissures according to visual CT analysis. $11(61 \%)$ of the patients responded well to valve treatment. Another publication [24] recommends the exclusion of collateral ventilation in the collapse phenomenon lobe through Chartis assessments of the ipsilateral adjacent lobes. When those lobes were collateral ventilation negative, the collapse phenomenon lobe was shown to benefit from valve insertion in nine (75\%) out of 12 patients.

In summary, the collapse phenomenon is seen in Chartis assessments more frequently under jet ventilation than spontaneous breathing and this pattern almost always occurs in the lower lobes. Its origin is still unclear. When confronted with the collapse phenomenon, collateral ventilation can be excluded through collateral ventilation negative assessment under spontaneous ventilation or collateral ventilation negative assessment of the ipsilateral adjacent lobes using Chartis or by CT fissure analysis in order to successfully treat collapse phenomenon lobes. Future studies will show if, and under which algorithm, Chartis and CT analysis can be complementary tools for collateral ventilation analysis.

\section{References}

1 Kohn HN. Zur Histologie der indurierenden fibrinösen Pneumonie. Münchener Medizinische Wochenschrift 1893; 40: 42-45.

2 Sciurba FC, Ernst A, Herth FJ, et al. A randomized study of endobronchial valves for advanced emphysema. N Engl J Med 2010; 363: 1233-1244.

3 Shah PL, Herth FJ. Current status of bronchoscopic lung volume reduction with endobronchial valves. Thorax 2014; 69: 280-286.

4 Valipour A, Herth FJ, Burghuber OC, et al. Target lobe volume reduction and COPD outcome measures after endobronchial valve therapy. Eur Respir J 2014; 43: 387-396.

5 Venuta F, Anile M, Diso D, et al. Long-term follow-up after bronchoscopic lung volume reduction in patients with emphysema. Eur Respir J 2012; 39: 1084-1089.

6 Herth FJ, Noppen M, Valipour A, et al. Efficacy predictors of lung volume reduction with Zephyr valves in a European cohort. Eur Respir J 2012; 39: 1334-1342.

7 Herzog D, Poellinger A, Doellinger F, et al. Modifying post-operative medical care after EBV implant may reduce pneumothorax incidence. PLoS One 2015; 10: e0128097.

8 Davey C, Zoumot Z, Jordan S, et al. Bronchoscopic lung volume reduction with endobronchial valves for patients with heterogeneous emphysema and intact interlobar fissures (the BeLieVeR-HIFi study): a randomised controlled trial. Lancet 2015; 386: 1066-1073. 
Gompelmann D, Eberhardt R, Slebos DJ, et al. Diagnostic performance comparison of the Chartis System and
high-resolution computerized tomography fissure analysis for planning endoscopic lung volume reduction. Respirology 2014; 19: 524-530.

31 Valipour A, Burghuber OC. An update on the efficacy of endobronchial valve therapy in the management of hyperinflation in patients with chronic obstructive pulmonary disease. Ther Adv Respir Dis 2015; 9: $294-301$.

32 West JB. Challenges in teaching the mechanics of breathing to medical and graduate students. Adv Physiol Educ 2008; 32: 177-184. 